\title{
Amination of Heterocyclic Compound with O-Benzoylhydroxylamine Derivatives
}

\author{
Luca Parlanti, Robert P. Discordia, John Hynes, Jr., ${ }^{\dagger}$ Michael M. Miller, ${ }^{\dagger}$ Harold R. \\ O'Grady, ${ }^{\dagger}$ Zhongping Shi*
}

Departments of Process Research and Development and Drug Discovery Chemistry, ${ }^{\dagger}$ Bristol-Myers Squibb Research \& Development, P.O. Box 4000, Princeton, New Jersey 08543

Zhongping.shi@bms.com

\section{Supporting Information}

\section{Experimental Section}

All starting material and reagents were commercial grade and used without further purification. Pyrrole $\mathbf{1 a},{ }^{1} \mathbf{1 b}^{2}$ and $\mathbf{1} \mathbf{d}^{3}$ were prepared according to the literature.

General Procedure for Amination: Diethyl 1-Amino-3-methylpyrrole-2,4dicarboxylate (3a). A solution of potassium t-butoxide $(1 \mathrm{M}, 110 \mathrm{~mL})$ was added to a solution of $1 \mathrm{a}(22.5 \mathrm{~g}, 10.0 \mathrm{~mol})$ in NMP $(130 \mathrm{~mL})$ at $20^{\circ} \mathrm{C}$. After $20 \mathrm{~min}$, a solution of aminating reagent $\mathbf{5 h}(11.5 \mathrm{~mol})$ in NMP $(80 \mathrm{~mL})$ was added at $<25^{\circ} \mathrm{C}$. The mixture was stirred at $20{ }^{\circ} \mathrm{C}$ for $20 \mathrm{~min}$. The conversion of BMS-529303 was determined by HPLC analysis. The reaction mixture was treated with brine $(7 \%, 400 \mathrm{~mL})$ and ethyl acetate $(400 \mathrm{~mL})$. The organic layer was separated and the aqueous layer was extracted with ethyl acetate $(400 \mathrm{~mL})$. The combined organic layers were washed with aqueous sodium bicarbonate solution $(5 \%, 300 \mathrm{~mL})$ and then water $(300 \mathrm{~mL})$. The organic solution was dried over anhydrous sodium sulfate and concentrated to give a crude 3a $(23.7 \mathrm{~g})$. The crude 3a was purified by flash chromatography on silica gel (elute with 5-15\% of ethyl acetate in heptane) to give $22.1 \mathrm{~g}(92 \%)$ of $3 \mathrm{a}^{4} \mathrm{mp} 57.0-59.0{ }^{\circ} \mathrm{C}$.

Ethyl 1-Amino-2-(n-propylcarboxamido)-3-methylpyrrole-4-carboxylate (3b). ${ }^{4} \quad 86 \%$ yield. $\mathrm{mp} 97.0-99.0^{\circ} \mathrm{C}$.

Ethyl 1-Amino-3,5-dimethylpyrrole-2-carboxylate (3c): $84 \%$ yield. oil. ${ }^{1} \mathrm{H}$ NMR $\left(\mathrm{CDCl}_{3}, 500 \mathrm{MHz}\right) \delta 5.66(\mathrm{~s}, 1 \mathrm{H}), 5.33\left(\mathrm{br} \mathrm{s}, 2 \mathrm{H}, \mathrm{NH}_{2}\right), 4.30(\mathrm{q}, 2 \mathrm{H}, J=7.5 \mathrm{~Hz}), 2.26(\mathrm{~s}$, $3 \mathrm{H}), 2.23(\mathrm{~s}, 3 \mathrm{H}), 1.36(\mathrm{t}, 3 \mathrm{H}, J=7.5 \mathrm{~Hz}) ;{ }^{13} \mathrm{C} \mathrm{NMR}\left(\mathrm{CDCl}_{3}, 125 \mathrm{MHz}\right) \delta 162.7,136.0$, 127.4, 116.9, 107.2, 59.4, 14.5, 14.3, 11.30; ES HRMS m/e $\mathrm{C}_{9} \mathrm{H}_{15} \mathrm{~N}_{2} \mathrm{O}_{2}(\mathrm{M}+\mathrm{H})^{+} \mathrm{Calcd}$ : 183.1128; Found: 183.1120; Elemental Analysis: Calcd. for $\mathrm{C}_{9} \mathrm{H}_{14} \mathrm{~N}_{2} \mathrm{O}_{2}$ : C $59.32 \%, \mathrm{H}$ 
7.74\%, N 15.37\%; Found: C 59,62\%, H 7.48\%, N 15.28\%. IR (KBr) 3335, 2926, 1678, $1485,1411,1252,1152,1091 \mathrm{~cm}^{-1}$.

Methyl 1-Amino-2-methyl-7-methoxyindole-3-carboxylate (3d). ${ }^{4} \quad 91 \%$ yield. mp $122.0-124.0{ }^{\circ} \mathrm{C}$.

Ethyl 1-Amino-2-methylindole-3-carboxylate (3e). ${ }^{4} \quad 88 \%$ yield. $\mathrm{mp} 94.0-96.0{ }^{\circ} \mathrm{C}$.

Ethyl 1-Amino-5-methoxyindole-2-carboxylate (3f). $82 \%$ yield. mp 108.0-110.0 ${ }^{\circ} \mathrm{C}$. ${ }^{1} \mathrm{H}$ NMR $\left(\mathrm{CDCl}_{3}, 400 \mathrm{MHz}\right) \delta 7.50(\mathrm{~d}, 1 \mathrm{H}, J=9.2 \mathrm{~Hz}), 7.06(\mathrm{~s}, 1 \mathrm{H}), 7.00(\mathrm{dd}, 1 \mathrm{H}, J=$ 9.2, $2.5 \mathrm{~Hz}), 6.97$ (d, 1H, $J=2.5 \mathrm{~Hz}$ ), 5.27 (br s, 2H, NH ), 4.35 (q, 2H, $J=7.0 \mathrm{~Hz}$ ), 3.82 $(\mathrm{s}, 3 \mathrm{H}), 1.39(\mathrm{t}, 3 \mathrm{H}, J=7.0 \mathrm{~Hz}) ;{ }^{13} \mathrm{C} \mathrm{NMR}\left(\mathrm{CDCl}_{3}, 100 \mathrm{MHz}\right) \delta 162.4,154.8,134.9$, 126.3, 123.1, 117.2, 111.8, 107.1, 102.0, 60.6, 55.7, 14.3. Elemental Analysis: Calcd. for $\mathrm{C}_{12} \mathrm{H}_{14} \mathrm{~N}_{2} \mathrm{O}_{3} \mathrm{C}$ 61.52, H 6.02, N 11.95\%; Found: C 61.76, H 5.98, N 11.79. IR (KBr) $3354,2980,1685,1519,1471,1451,1214,1147,1098,1030 \mathrm{~cm}^{-1}$.

1-Amino-4,5-diphenylimidazole (3h). $\quad 80 \%$ yield. mp. 195.0-197.0 ${ }^{\circ} \mathrm{C} . \quad{ }^{1} \mathrm{H}$ NMR (DMSO-d6, $400 \mathrm{MHz}) \delta 7.98(\mathrm{~s}, 1 \mathrm{H}), 7.38-7.50(\mathrm{~m}, 5 \mathrm{H}), 7.38(\mathrm{dd}, 2 \mathrm{H}, J=8.3,1.4 \mathrm{~Hz})$, $7.22(\mathrm{dd}, 2 \mathrm{H}, J=8.3,7.5 \mathrm{~Hz}), 7.15(\mathrm{dt}, 1 \mathrm{H}, J=7.5,1.3 \mathrm{~Hz}), 5.98$ (br s, $\left.2 \mathrm{H}, \mathrm{NH}_{2}\right) .{ }^{13} \mathrm{C}$ NMR (DMSO-d6, 100 MHz) $\delta 137.0(\mathrm{CH}), 133.7,133.5,130.5(2 \mathrm{C}), 129.0,128.8,128.3$ (2C), 128.1, 127.9 (2C), 126.3, 126.1 (2C). ES HRMS m/e $\mathrm{C}_{9} \mathrm{H}_{11} \mathrm{~N}_{3}(\mathrm{M}+\mathrm{H})^{+}$Calcd: 236.1186; Found: 236.1181. Elemental Analysis: Calcd. for $\mathrm{C}_{15} \mathrm{H}_{13} \mathrm{~N}_{3}$ : C 76.57, H 5.57, N 17.86; Found: C 76.27, H 5.67, N, 17.83. IR $\left(\mathrm{KBr}, \mathrm{cm}^{-1}\right) 3348,3117,2973,1646$, 1599, 1504, 1440, 1068, 951, 916, 771, 739, 698.

1-Amino-5,6-dimethylbenzimidazole (3i). $84 \%$ yield. mp. $230.0-232.0{ }^{\circ} \mathrm{C} ;{ }^{1} \mathrm{H}$ NMR (DMSO-d6, $400 \mathrm{MHz}) \delta 7.91$ (s, 1H), 7.35 (s, 1H), 7.27 (s, 1H), 6.03 (s, 2H, NH ), 2.33 (s, 3H), $2.29(\mathrm{~s}, 3 \mathrm{H}) .{ }^{13} \mathrm{C}$ NMR (DMSO-d6, $\left.100 \mathrm{MHz}\right) \delta 143.5,140.0,133.4,130.6$, 129.5, 119.4, 109.9, 20.1, 19.8. ES HRMS m/e $\mathrm{C}_{9} \mathrm{H}_{11} \mathrm{~N}_{3}(\mathrm{M}+\mathrm{H})^{+}$Calcd: 162.1031; Found 162.1033. Elemental Analysis: Calcd. for $\mathrm{C}_{9} \mathrm{H}_{11} \mathrm{~N}_{3}$ : C 67.05, H 6.87, N 26.06; Found: C 67.01, H 7.05, N 25.86. IR (KBr, cm $\left.{ }^{-1}\right) 3306,3170,3079,2974,1637,1492$, $1468,1386,1327,1253,1049,985,904,849$.

Diethyl 1-Amino-pyrazole-3,5-dicarboxylate (3j). 95\% yield. $\mathrm{mp} 61.0-63.0{ }^{\circ} \mathrm{C} .{ }^{1} \mathrm{H}$ NMR $\left(\mathrm{CDCl}_{3}, 400 \mathrm{MHz}\right) \delta 7.23(\mathrm{~s}, 1 \mathrm{H}), 6.24($ br s, 2H, NH$), 4.355(\mathrm{q}, 2 \mathrm{H}, J=7.0 \mathrm{~Hz})$, $4.350(\mathrm{q}, 2 \mathrm{H}, J=7.0 \mathrm{~Hz}), 1.357(\mathrm{t}, 3 \mathrm{H}, J=7.0 \mathrm{~Hz}), 1.353(\mathrm{t}, 3 \mathrm{H}, J=7.0 \mathrm{~Hz}) .{ }^{13} \mathrm{C} \mathrm{NMR}$ $\left(\mathrm{CDCl}_{3}, 100 \mathrm{MHz}\right) \delta 161.2,159.8,137.5,127.6,111.8,61.8,61.1,14.2,14.1$. ES HRMS $m / e \mathrm{C}_{9} \mathrm{H}_{14} \mathrm{~N}_{3} \mathrm{O}_{4}(\mathrm{M}+\mathrm{H})^{+}$Calcd. 228.0979; Found 228.0980. IR (KBr) 3333, 2983, 1734, $1282,1220,1111,1081,1026,759 \mathrm{~cm}^{-1}$.

9-Amino-3,6-dibromocarbazole (3k). $\quad 87 \%$ yield. $\mathrm{mp} \quad 204.0-208.0{ }^{\circ} \mathrm{C} .{ }^{1} \mathrm{H}$ NMR (DMSO-d6, $400 \mathrm{MHz}) \delta 8.43(\mathrm{~d}, 2 \mathrm{H}, J=2.0 \mathrm{~Hz}), 7.62(\mathrm{dd}, 2 \mathrm{H}, J=8.8,2.0 \mathrm{~Hz}), 7.56(\mathrm{~d}$, $2 \mathrm{H}, J=8.8$ ), 5.95 (br s, $2 \mathrm{H}, \mathrm{NH}_{2}$ ). ${ }^{13} \mathrm{C}$ NMR (DMSO-d6, $\left.100 \mathrm{MHz}\right) \delta 140.1,128.7$, 123.1, 120.5, 111.3, 111.0. Elemental Analysis: Calcd. for $\mathrm{Cl}_{2} \mathrm{H}_{8} \mathrm{Br}_{2} \mathrm{~N}_{2}$ : C 42.39, $\mathrm{H}$ 2.37, 
N 8.23, Br 47.00; Found: C 42.03, H 2.17, N 8.13, Br 45.90. IR (KBr) 3340, 1466, 1434, $1286,1236,1048,799 \mathrm{~cm}^{-1}$.

Preparation of $\boldsymbol{O}$-4-Nitrobenzoylhydroxylamine (5a). ${ }^{5} \quad$ Triethylamine $(40.7 \mathrm{~g}, 402$ mmol) was added to a solution of N-Boc-hydroxyamine $(50.0 \mathrm{~g}, 368 \mathrm{mmol})$ in $200 \mathrm{~mL}$ of dichloromethane at room temperature. The solution was cooled to $0{ }^{\circ} \mathrm{C}$. A solution of 4nitrobenzoyl chloride $(69.1 \mathrm{~g}, 365 \mathrm{mmol})$ in dichloromethane $(320 \mathrm{~mL})$ was slowly added at $0-5{ }^{\circ} \mathrm{C}$ over $65 \mathrm{~min}$. The mixture was stirred at $0-5{ }^{\circ} \mathrm{C}$ for $5 \mathrm{~min}$ and then warmed up to $15-20{ }^{\circ} \mathrm{C}$. Water $(300 \mathrm{~mL})$ was added to quench the reaction. After stirring for $5 \mathrm{~min}$, the organic layer was separated, washed with aqueous $\mathrm{K}_{2} \mathrm{HPO}_{4}(4 \%, 200 \mathrm{~mL})$, and then treated with methanesulfonic acid $(52.6 \mathrm{~g}, 548 \mathrm{mmol})$ at $25^{\circ} \mathrm{C}$ for $20 \mathrm{~h}$. The mixture was treated with aqueous $\mathrm{K}_{2} \mathrm{HPO}_{4}(20 \%, 610 \mathrm{~g})$ at $25{ }^{\circ} \mathrm{C}$ for $5-10 \mathrm{~min}$. Tetrahydrofuran (520 $\mathrm{mL}$ ) was added to the mixture. The organic layer was separated, washed with brine $(25 \%, 530 \mathrm{~g})$, and dried over $\mathrm{Na}_{2} \mathrm{SO}_{4}(50 \mathrm{~g})$ for 30 minutes. After removal of $\mathrm{Na}_{2} \mathrm{SO}_{4}$ by filtration, the filtrate was concentrated under vacuum at $25-35{ }^{\circ} \mathrm{C}$ to about $355 \mathrm{~g}$. Heptane $(440 \mathrm{~mL})$ was slowly added to the mixture for crystallization. The solid was collected by filtration, washed with THF-heptane $(1: 2,90 \mathrm{~mL})$, and dried under vacuum at $25-35{ }^{\circ} \mathrm{C}$ to give a slightly yellow product 5a $(56.6 \mathrm{~g}, 85 \%, 99.2 \mathrm{AP}) . \quad \mathrm{mp} 85.0{ }^{\circ} \mathrm{C}$ (dec., lit $\left.^{5}, 111.5^{\circ} \mathrm{C}\right) .{ }^{1} \mathrm{H}$ NMR $\left(\mathrm{CDCl}_{3}, 400 \mathrm{MHz}\right) \delta 8.32(\mathrm{~d}, 2 \mathrm{H}, J=8.4 \mathrm{~Hz}), 8.21(\mathrm{~d}, 2 \mathrm{H}$, $J=8.4 \mathrm{~Hz}), 6.76\left(\mathrm{~s}, 2 \mathrm{H}, \mathrm{NH}_{2}\right) .{ }^{13} \mathrm{C} \mathrm{NMR}\left(\mathrm{CDCl}_{3}, 100 \mathrm{MHz}\right) \delta 165.5,150.8,133.3$, 130.6 (2C), 123.7 (2C). Elemental Analysis: Calcd. for $\mathrm{C}_{7} \mathrm{H}_{6} \mathrm{~N}_{2} \mathrm{O}_{4}: \mathrm{C} 46.16, \mathrm{H} 3.32, \mathrm{~N}$ 15.38; Found: C 46.15, H 3.14, N 15.34.

Aminating reagents $\mathbf{5 b}$-e and $\mathbf{5 h}-\mathbf{I}$ were prepared in the same wayas above, except for that dichloromethane, instead of tetrahydrofuran, was used for the extractive work up and crystallization with heptane.

O-4-Fluorobenzoylhydroxylamine (5b). $84 \%$ yield. mp $37.0-39.0{ }^{\circ} \mathrm{C}$ (dec.). ${ }^{1} \mathrm{H} \mathrm{NMR}$ $\left(\mathrm{CDCl}_{3}, 400 \mathrm{MHz}\right) \delta 8.02(\mathrm{dd}, 2 \mathrm{H}, J=8.8,5.7 \mathrm{~Hz}), 7.11(\mathrm{t}, 2 \mathrm{H}, J=8.8 \mathrm{~Hz}), 6.61$ (br s, $\left.2 \mathrm{H}, \mathrm{NH}_{2}\right) .{ }^{13} \mathrm{C} \mathrm{NMR}\left(\mathrm{CDCl}_{3}, 100 \mathrm{MHz}\right) \delta 166.6,166.0(\mathrm{~d}, J=255 \mathrm{~Hz}), 132.0(\mathrm{~d}, 2 \mathrm{C}, J=$ $10.2 \mathrm{~Hz}), 124.2,115.8(\mathrm{~d}, 2 \mathrm{C}, J=22.3 \mathrm{~Hz})$. Elemental Analysis: Calcd. for $\mathrm{C}_{7} \mathrm{H}_{6} \mathrm{FNO}_{2}$ : C 54.20, H 3.90, F 12.25, N 9.03; Found: C 53.94, H 3.88, F 12.61, N 8.79. IR (KBr, cm 1) 3299, 2300-3300, 1698, 1655, 1620, 1605, 1582, 1508, 1244, 1157, 849 .

O-3-Chlorobenzoylhydroxylamine (5c). $77 \%$ yield. mp $57.0-59.0{ }^{\circ} \mathrm{C}\left(\right.$ lit $^{6}, 51-52{ }^{\circ} \mathrm{C}$ dec). ${ }^{1} \mathrm{H} \mathrm{NMR}\left(\mathrm{CDCl}_{3}, 400 \mathrm{MHz}\right) \delta 7.98(\mathrm{dd}, 1 \mathrm{H}, J=2.2,1.8 \mathrm{~Hz}), 7.88(\mathrm{br} \mathrm{d}, 1 \mathrm{H}, J=$ $7.9 \mathrm{~Hz}$ ), 7.55 (br d, $1 \mathrm{H}, J=7.9 \mathrm{~Hz}), 7.39$ (t, $1 \mathrm{H}, J=7.9 \mathrm{~Hz}), 6.62$ (br s, $\left.2 \mathrm{H}, \mathrm{NH}_{2}\right) .{ }^{13} \mathrm{C}$ NMR $\left(\mathrm{CDCl}_{3}, 100 \mathrm{MHz}\right) \delta 166.3,134.8,133.5,129.9,129.7,129.5,127.5$. Elemental Analysis: Calcd. for $\mathrm{C}_{7} \mathrm{H}_{6} \mathrm{ClNO}_{2}$ : C 49.00, H 3.52, Cl 20.66, N 8.16; Found: C 49.05, H 3.38, Cl 20.79, N 8.15. IR (KBr, cm $\left.{ }^{-1}\right)$ 3297, 3218, 3144, 1720, 1601, 1575, 1426, 1297 , 1267, 1219, 1073, 862, 739 .

O-4-Chlorobenzoylhydroxylamine (5d). $89 \%$ yield. mp 81.0-83.0 ${ }^{\circ} \mathrm{C}$ (dec.). ${ }^{1} \mathrm{H} \mathrm{NMR}$ $\left(\mathrm{CDCl}_{3}, 400 \mathrm{MHz}\right) \delta 7.93(\mathrm{~d}, 2 \mathrm{H}, J=8.8 \mathrm{~Hz}), 7.41(\mathrm{~d}, 2 \mathrm{H}, J=8.8 \mathrm{~Hz}), 6.61$ (br s, 2H, $\left.\mathrm{NH}_{2}\right) .{ }^{13} \mathrm{C} \mathrm{NMR}\left(\mathrm{CDCl}_{3}, 100 \mathrm{MHz}\right) \delta 166.7,140.0,130.7$ (2C), 128.9 (2C), 126.4. Elemental Analysis: Calcd. for $\mathrm{C}_{7} \mathrm{H}_{6} \mathrm{ClNO}_{2}$ : C 49.00, $\mathrm{H} 3.52, \mathrm{Cl} 20.66, \mathrm{~N} 8.16$; Found: $\mathrm{C}$ 49.29, H 3.41, Cl 20.90, N 7.88. IR (KBr, cm $\left.{ }^{-1}\right) 3305,1732,1596,1488,1401,1271$, 1094, 1073, 1012, 849, 754. 
O-4-Bromobenzoylhydroxylamine (5e). $82 \%$ yield. $\mathrm{mp} 88.0-90.0{ }^{\circ} \mathrm{C}$ (dec.). ${ }^{1} \mathrm{H}$ NMR $\left(\mathrm{CDCl}_{3}, 400 \mathrm{MHz}\right) \delta 7.88(\mathrm{~d}, 2 \mathrm{H}, J=8.8 \mathrm{~Hz}), 7.60(\mathrm{~d}, 2 \mathrm{H}, J=8.8 \mathrm{~Hz}), 6.64(\mathrm{~s}, 2 \mathrm{H}$, $\left.\mathrm{NH}_{2}\right) .{ }^{13} \mathrm{C} \mathrm{NMR}\left(\mathrm{CDCl}_{3}, 100 \mathrm{MHz}\right) \delta 166.8,132.0$ (2C), 130.9 (2C), 128.7, 126.9. Elemental Analysis: Calcd. for $\mathrm{C}_{7} \mathrm{H}_{6} \mathrm{BrNO}_{2}$ : C 38.91, $\mathrm{H}$ 2.80, Br 36.98, N 6.48; Found: C 38.95, H 2.85, Br 37.67, N 6.46. IR (KBr, $\left.\mathrm{cm}^{-1}\right)$ 3284, 3217, 1729, 1588, 1397, 1290, 1277, 1071, 1010, 844, 754.

$\boldsymbol{O}$-4-Methoxybenzoylhydroxylamine (5h). $\quad 89 \%$ yield. $\mathrm{mp} \quad 34.0-35.0{ }^{\circ} \mathrm{C} .{ }^{1} \mathrm{H}$ NMR $\left(\mathrm{CDCl}_{3}, 400 \mathrm{MHz}\right) \delta 7.97(\mathrm{~d}, 2 \mathrm{H}, J=8.8 \mathrm{~Hz}), 6.93(\mathrm{~d}, 2 \mathrm{H}, J=8.8 \mathrm{~Hz}), 6.58(\mathrm{br} \mathrm{s}, 2 \mathrm{H}$, $\left.\mathrm{NH}_{2}\right), 3.86(\mathrm{~s}, 3 \mathrm{H}) .{ }^{13} \mathrm{C} \mathrm{NMR}\left(\mathrm{CDCl}_{3}, 100 \mathrm{MHz}\right) \delta 167.4,163.8,131.5(2 \mathrm{C}), 120.3$, 113.9 (2C), 55.5. Elemental Analysis: Calcd. for $\mathrm{C}_{8} \mathrm{H}_{9} \mathrm{NO}_{3}$ : C 57.48, $\mathrm{H}$ 5.42, $\mathrm{N} 8.38$; Found: C 57.43, H 5.24, N 8.16. IR $\left(\mathrm{KBr}, \mathrm{cm}^{-1}\right)$ 3285, 2300-3300, 1687, 1643, 1606, $1569,1508,1428,1303,1256,1166,1024,846$.

O-2,4-Dimethoxybenzoylhydroxylamine (5i). $86 \%$ yield. $\mathrm{mp} 66.0-67.0{ }^{\circ} \mathrm{C} .{ }^{1} \mathrm{H} \mathrm{NMR}$ $\left(\mathrm{CDCl}_{3}, 400 \mathrm{MHz}\right) \delta 7.84(\mathrm{~d}, 1 \mathrm{H}, J=8.8 \mathrm{~Hz}), 6.54\left(\mathrm{~s}, 2 \mathrm{H}, \mathrm{NH}_{2}\right), 6.51(\mathrm{dd}, 1 \mathrm{H}, J=8.8$, $2.2 \mathrm{~Hz}), 6.49(\mathrm{~d}, 1 \mathrm{H}, J=2.2 \mathrm{~Hz}), 3.89(\mathrm{~s}, 3 \mathrm{H}), 3.86(\mathrm{~s}, 3 \mathrm{H}) .{ }^{13} \mathrm{C} \mathrm{NMR}\left(\mathrm{CDCl}_{3}, 100\right.$ $\mathrm{MHz}) \delta 167.0,164.8,161.3,133.7,110.0,104.9,99.0,56.0,55.6$. Elemental Analysis: Calcd. for $\mathrm{C}_{9} \mathrm{H}_{11} \mathrm{NO}_{4}$ : C 54.82, H 5.62, N 7.10; Found: C 54.83, H 5.71, N 7.09. IR $\left(\mathrm{KBr}, \mathrm{cm}^{-1}\right) 3304,3250,2999,1724,1614,1574,1465,1274,1260,1161,1056,1027$ 829,762 .

1. Hamamoto, I. Jpn. Kokai Tokkyo Koho (1996), 6 pp. JP 08059611 A2 19960305 Heisei. CAN 125:10608 AN 1996:326165.

2. 1b was prepared by EDCI/HOBt-mediated coupling of $n$-propylamine with 3methylpyrrole-2,4-dicarboxylic acid-4-ethyl ester. See: Crowin, A. H.; Viohl, P. J. Am. Chem. Soc. 1944, 66, 1137.

3. Hynes, J., Jr.; Leftheris, K.; Wu, H.; Pandit, C.; Chen, P.; Norris, D. J.; Chen, B.C.; Zhao, R.; Kiener, P. A.; Chen, X.; Turk, L. A.; Patil-Koota, V.; Gillooly, K. M.; Shuster, D. J.; McIntyre, K. W. Bioorg. Med. Chem. Lett. 2002, 12, 2399.

4. Hynes, J.; Doubleday, W.; Dyckman, A.; Godfrey, J.; Grosso, J.; Kiau, S.; Leftheris, K. J. Org. Chem. 2004, 69, 1368.

5. This is an improved process based on a literature for preparation of $\mathbf{5 f}$ : Carpino, L.; Giza, C.; Carpino, B. J. Am. Chem. Soc. 1959, 81, 955.

6. Marmer, W.; Maerker, G. J. Org. Chem. 1972, 37, 3520. 